\title{
Dark Future for Dark Matter
}

\author{
Donald H. Eckhardt ${ }^{1}$, José Luis Garrido Pestaña ${ }^{2 *}$ \\ ${ }^{1}$ Canterbury, NH, USA \\ ${ }^{2}$ Departamento de Física, Universidad de Jaén, Campus Las Lagunillas, Jaén, España \\ Email: ${ }^{*} l g @ u j a e n . e s$
}

How to cite this paper: Eckhardt, D.H. and Garrido Pestaña, J.L. (2020) Dark Future for Dark Matter. Journal of Modern Physics, 11, 1589-1597.

https://doi.org/10.4236/jmp.2020.1110099

Received: September 2, 2020

Accepted: October 17, 2020

Published: October 20, 2020

Copyright $\odot 2020$ by author(s) and Scientific Research Publishing Inc. This work is licensed under the Creative Commons Attribution International License (CC BY 4.0).

http://creativecommons.org/licenses/by/4.0/

\begin{abstract}
The prevailing cosmological constant and cold dark matter $(\Lambda \mathrm{CDM})$ cosmic concordance model accounts for the radial expansion of the universe after the Big Bang. The model appears to be authoritative because it is based on the Einstein gravitational field equation. However, a thorough scrutiny of the underlying theory calls into question the suitability of the field equation, which states that the Einstein tensor $\boldsymbol{G}_{\mu \nu}$ is a constant multiple of the stress-energy tensor $\boldsymbol{T}_{\mu \nu}$ when they both are evaluated at the same $4 \mathrm{D}$ space-time point: $\boldsymbol{G}_{\mu \nu}=8 \pi k \boldsymbol{T}_{\mu \nu}$, where $k$ is the gravitational constant. Notwithstanding its venerable provenance, this equation is incorrect unless the cosmic pressure is $p=0$; but then all that remains of the Einstein equation is the Poisson equation which models the Newtonian gravity field. This shortcoming is not resolved by adding the cosmological constant term to the field equation, $\boldsymbol{G}_{\mu v}+\Lambda \boldsymbol{g}_{\mu \nu}=8 \pi k \boldsymbol{T}_{\mu \nu}$, as in the $\Lambda \mathrm{CDM}$ model, because then $p=\Lambda$, so the pressure is a universal constant, not a variable. Numerous studies support the concept of a linearly expanding universe in which gravitational forces and accelerations are negligible because the baryonic mass density of the universe is far below its critical density. We show that such a coasting universe model agrees with $\mathrm{SNe}$ Ia luminosity vs. redshift distances just as well or even better than the $\Lambda \mathrm{CDM}$ model, and that it does so without having to invoke dark matter or dark energy. Occam's razor favors a coasting universe over the $\Lambda$ CDM model.
\end{abstract}

\section{Keywords}

Classical General Relativity, General Relativity and Gravitation:

Fundamental Problems and General Formalism, Fundamental

Aspects of Astrophysics, Relativity and Gravitation,

Dark Energy, Mathematical and Relativistic

Aspects of Cosmology 


\section{Introduction}

The $\Lambda C D M$ model is used to represent the expanding universe for the interpretation 1) of type Ia supernovae (SNe Ia) brightness and redshift data, and 2), of measurements of anisotropies in the cosmic microwave background (CMB) spectrum of the early universe. The model conjectures the existence of dark matter and dark energy - a lot of both. With a felicitous balance of the two, an ostensibly credible model is derived. However, a $\Lambda$ CDM model has drawbacks. Firstly, the existence of dark matter is an unverified hypothesis. ${ }^{1}$ There have been numerous experiments dedicated to finding dark matter here on Earth, in underground experiments [2] [3] and satellite detectors [4], as well as in the Large Hadron Collider [5]. Yet, there has been no unambiguous dark matter detection [6], which, by induction [7], is evidence of absence. Moreover, aside from the $\Lambda \mathrm{CDM}$ model, there is no theoretical call for dark energy; nor has there even been an observational hint of its existence. Kang et al. [8] have recently challenged a key premise in the derivation of the $\Lambda$ CDM model, and by addressing the problem with a different approach, we show that the model is utterly untenable. We expand, revise and interpret the Einstein field equation to find a simple viable alternative to the $\Lambda \mathrm{CDM}$ model.

\section{Einstein Field Equation}

We use the standard nomenclature of GR (e.g., see Kenyon [9]), except $k$ is the Newtonian gravitational constant and $c=1$ is the speed of light. The Einstein field equation is

$$
\boldsymbol{G}_{\mu v}=8 \pi k \boldsymbol{T}_{\mu v}
$$

where $\boldsymbol{G}_{\mu \nu}$ is the Einstein tensor and $\boldsymbol{T}_{\mu \nu}$ is the stress-energy tensor of a perfect fluid,

$$
\boldsymbol{T}_{\mu v}=\{\{\rho, p, p, p\}\},
$$

where $\{\{\cdots\}\} \equiv \operatorname{diag}[\cdots], \quad \rho$ is the mass density, and $p$ is the pressure (energy density).

The line element, $\mathrm{d} s^{2}=\boldsymbol{g}_{\mu \nu} \mathrm{d} x^{\mu} \mathrm{d} x^{\nu}$, reduces for the weak isotropic cosmic field, to ([10], Eq. 10.84)

$$
\mathrm{d} s^{2}=(1+2 \phi) \mathrm{d} t^{2}-(1-2 \phi) \delta_{i j} \mathrm{~d} x^{i} \mathrm{~d} x^{j},
$$

According to Einstein ([11], p. 84), $\boldsymbol{G}_{\mu v}$ "must be a differential tensor in the $\boldsymbol{g}_{\mu v}$ that is completely determined by the following three conditions:

1) It may contain no differential coefficients of the $\boldsymbol{g}_{\mu v}$ higher than the second.

${ }^{1}$ Dark matter had a sullied reputation from the nineteenth century, when astronomers searched fruitlessly for another sort of invisible matter-an intramercurial planet, Vulcan [1], that was thought to be responsible for otherwise unexplainable orbital perturbations of Mercury. As it turned out, what had been missing was not matter, but an authoritative theory: GR. So, is it now missing matter, or missing theory? 
2) It must be linear in these second differential coefficients.

3) Its divergence must vanish identically."

For the nonce, let $\partial$ represent any partial derivative operator $\partial_{x^{i}}$, and let $g$ represent any component of $\boldsymbol{g}_{\mu v}$. According to the first two conditions, each component of $\boldsymbol{G}_{\mu v}$ has the form $\partial g \partial g \sim \partial \phi \partial \phi$ or $g \partial^{2} g \sim(1-2 \phi) \partial^{2} \phi \quad(\ldots$ or zero). Then, to the first power in $\phi$, the only non-zero form of a $\boldsymbol{G}_{\mu v}$ component is $\partial^{2} \phi$.

With $G_{00}=2 \partial^{2} \phi$ and $T_{00}=\rho$, Equation (1) yields the Poisson equation. If any other $\boldsymbol{G}_{\mu \nu}$ component, say $G_{11}$, is non-zero, then $G_{11} \sim \partial^{2} \phi=G_{00} / 2$; therefore $T_{11} \sim \rho$, and $G_{11} \neq 0$ implies that $p \sim \rho$. With such an equation of state, the fluid is relativistic, which is to say that the characteristic thermal speed of the fluid is close to $c$, which it generally is not. The only viable alternative is to set $G_{11}$ and all other terms of $\boldsymbol{G}_{\mu \nu}$ except $G_{00}$ equal to zero. But this requires that $p=0$, which is not generally true. We overcome this dilemma by adding $8 \pi k \boldsymbol{g}_{\mu \nu} p$ to the RHS of Equation (1). Then both sides of

$$
\boldsymbol{G}_{\mu v}=8 \pi k\left[\boldsymbol{T}_{\mu v}+\boldsymbol{g}_{\mu v} p\right]
$$

remain generally covariant and, with this essential correction, Equation (4) becomes

$$
\boldsymbol{G}_{\mu v}=\left\{\left\{2 \partial^{2} \phi, 0,0,0\right\}\right\}=8 \pi k\{\{\varrho, 0,0,0\}\},
$$

where $\varrho=\rho+p$. This is the full Poisson equation,

$$
\partial^{2} \phi=4 \pi k \varrho,
$$

which applies in all coordinate systems. It notably takes into account the gravitational charge equivalence of mass density $\rho$ and energy density $p[12] ;^{2}$ adapted to this nicety, $\phi$ is the Newtonian potential.

In general, the density does not need to be uniform. We can approximate the mass of the universe with an assemblage of point masses and consider just one point mass at a time. Then the Poisson equation for the potential due to one point mass $M$ is $\partial^{2} \phi=k M$, and the Einstein field equation is a linear superposiition of Poisson equations.

\section{The Expanding Universe}

For a universe with vanishingly small $\varrho$, the line element 3 reduces to

$$
\mathrm{d} s^{2}=\mathrm{d} t^{2}-\delta_{i j} \mathrm{~d} x^{i} \mathrm{~d} x^{j} .
$$

Then $\boldsymbol{G}_{\mu \nu}=0$ because this metric is independent of $\phi$; GR obviously does not apply. But what if this nearly empty universe expands according to Hubble's law? Then

$$
\mathrm{d} s^{2}=\mathrm{d} t^{2}-\mathrm{e}^{2 H_{0} t} \delta_{i j} \mathrm{~d} x^{i} \mathrm{~d} x^{j},
$$

${ }^{2}$ Using an arbitrary equation of state for a perfect fluid, Peebles ([11], Eq. 4.21) generalizes the RHS of Equation (6) to $4 \pi k \varrho \rightarrow 4 \pi k(\rho+3 p)$ instead of $4 \pi k \varrho \rightarrow 4 \pi k(\rho+p)$. 
so that $\boldsymbol{G}_{\mu \nu}=3 H_{0}^{2} \boldsymbol{g}_{\mu \nu}$, assuming that GR does apply. ${ }^{3}$ The Einstein field equation is $3 H_{0}^{2} \boldsymbol{g}_{\mu v}=0$, so $H_{0}=0$. Clearly GR does not apply in this case either. However, we can force GR to apply by replacing the Einstein tensor $\boldsymbol{G}_{\mu v}$ with its Lovelock [15] form $\boldsymbol{G}_{\mu \nu} \rightarrow \boldsymbol{G}_{\mu \nu}+b \boldsymbol{g}_{\mu \nu}$. Then the field equation is

$$
\left(3 H_{0}^{2}+b\right) \boldsymbol{g}_{\mu \nu}=0 .
$$

But $b$ is merely an invention that allows us to broaden the scope of the field equation by allowing for Hubble expansion; it has no significance of its own. In fact, the Hubble expansion is real, but it is independent of GR.

Refashioned to try to accommodate Hubble expansion, Equation (5) becomes

$$
\boldsymbol{G}_{\mu \nu}+b \boldsymbol{g}_{\mu \nu}=\left\{\left\{2 \partial^{2} \phi, 0,0,0\right\}\right\}+\Lambda \boldsymbol{g}_{\mu \nu}=8 \pi k\{\{\varrho, 0,0,0\}\},
$$

where $\Lambda=3 H_{0}^{2}+b$, which is zero according to Equation (9). There is no real change from Equation (5), but we use this form of the equation in Section 5, where $\Lambda$-there designated as the cosmological constant-is regarded as a proxy for dark energy.

\section{The Coasting Universe}

Evidence for the existence of dark matter is not at all conclusive. It is not needed to explain the flat rotation curves of spiral galaxies nor the CMB simulations. Concerning the rotation curves, alternate explanations have been published by Milgrom [16] [17], Brownstein and Moffat [18], Mannheim and O'Brien [19], Verlinde [20], ourselves [21], and others. As far as the CMB data are concerned, they show a compatibility with the $\Lambda \mathrm{CDM}$ model, but not that an equivalent compatibility is impossible for other models [22] [23] [24] [25]. Therefore if there is no dark matter $\varrho \approx \rho_{b}$, the density of baryonic matter, whose value [26], $\rho_{b} \approx 3.6 \times 10^{-28} \mathrm{~kg} \cdot \mathrm{m}^{-3}$, is two orders of magnitude smaller than the critical density, $\rho_{c}=3 H_{0}^{2} / 8 \pi k$, so the gravitational forces and accelerations are negligible. Kolb [27] proposed such a linearly coasting universe, which Sethi et al. [28] found to agree "surprisingly" well with SNe Ia observations. Subsequently many others [29]-[58] have confirmed the validity of a coasting fit, which we now corroborate. The coasting universe expands without acceleration,

$$
a(t)=1+H_{0} t .
$$

For redshift $z$, the distance between a source (then) and observer at $t=0$ (now) is

$$
r=\left(c / H_{0}\right) \ln (1+z) .
$$

The distance modulus depends on the area, $4 \pi f^{2}$, of the wavefront at $t=0$. For an open universe [59],

${ }^{3}$ We derive this expression using L. Parker's Mathematica [13] notebook, Curvature and the Einstein Equation [14], with only the following principal modifications:

coord $=\{\mathrm{t}, \mathrm{x}, \mathrm{y}, \mathrm{z}\}$;

g11 = $-\operatorname{Exp}[2 \mathrm{H} 0 \mathrm{t}]$

metric $=\{\{1,0,0,0\},\{0, \mathrm{~g} 11,0,0\},\{0,0, \mathrm{~g} 11,0\},\{0,0,0, \mathrm{~g} 11\}\}$ 


$$
f=\frac{c}{H_{0}} \sinh [\ln (1+z)]=\frac{c}{2 H_{0}}\left[1+z-\frac{1}{1+z}\right]
$$

is the luminosity distance.

In Figure 1, we plot a set of high-confidence Union 2.1 data [60]. We used these data to estimate $H_{0}=68.22 \pm 1.00 \mathrm{~km} \cdot \mathrm{s}^{-1} \cdot \mathrm{Mpc}^{-1}\left(\chi^{2}=576.45\right)$. It is the only parameter needed for calculating the superposed coasting curve. Because the number of degrees of freedom $v=579$ is large, the variable $\xi=\left(\chi^{2}-v\right) / \sqrt{2 v}$ is approximately normally distributed with unit variance. For our solution, $\xi=-0.075$ which, being a small value, substantiates the Union 2.1 observational error estimates as well as the coasting fit to the data. ${ }^{4}$ According to our $H_{0}$, the age of the coasting universe is the Hubble time, $H_{0}^{-1}=14.33 \pm 0.21 \mathrm{Gyr}$. This is gratifyingly close to the $14.46 \pm 0.31 \mathrm{Gyr}$ age of HD 140283, the oldest star for which a reliable age has been determined [62].

\section{The $\Lambda$ CDM Model}

Equating the $\mu v=00$ element pairs on both sides of Equation (10) gives

$$
2 \partial^{2} \phi+\Lambda=8 \pi k \varrho,
$$

whereas equating any of the $\mu v=11,22$ or 33 element pairs gives

$$
\Lambda=0 .
$$

Hubble diagram

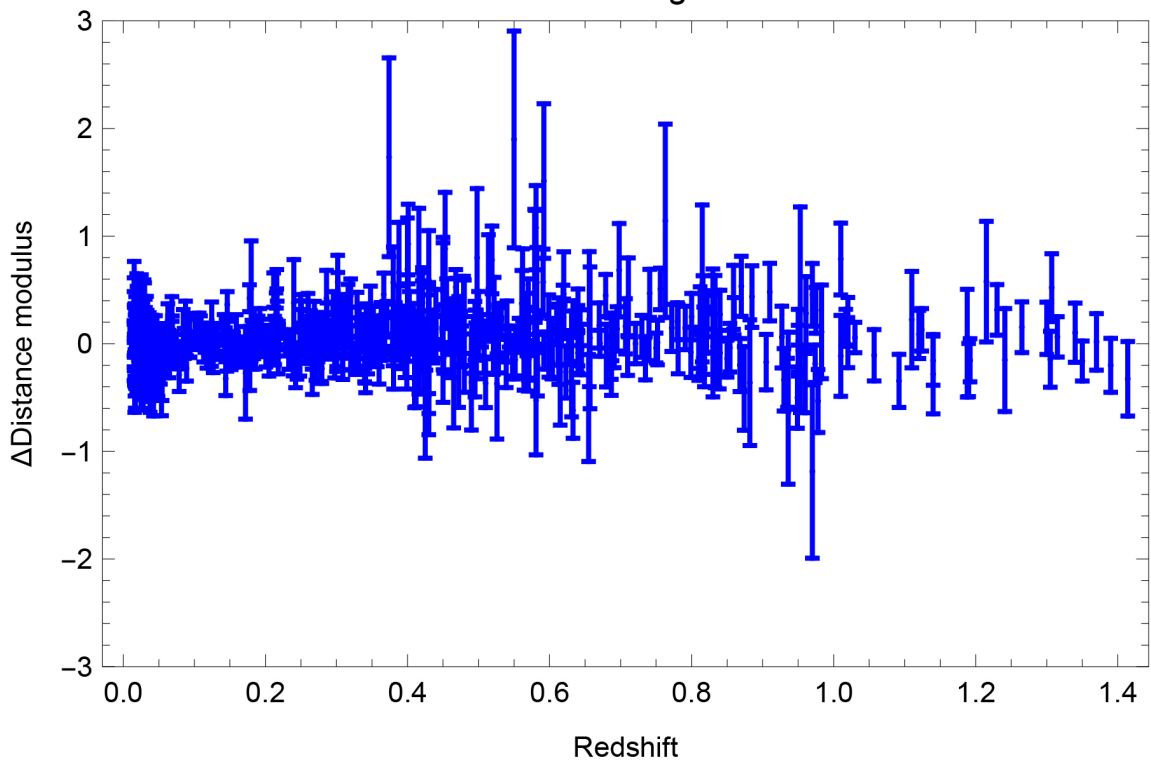

Figure 1. Hubble diagram residuals for the linearly coasting model that best fits the Union 2.1 compilation set of $580 \mathrm{SNe}$ Ia data points.

${ }^{4}$ With a three parameter $\left(H_{0}, \rho, \Lambda\right) \Lambda \mathrm{CDM}$ fit to the same Union 2.1 data, the Planck team [61] estimates $H_{0}=70 \mathrm{~km} \cdot \mathrm{s}^{-1} \cdot \mathrm{Mpc}^{-1}, \chi^{2}=545.11$, and $\xi=-0.939$, which we view per se as reasonable because the magnitude of $\xi$, although larger than ours, is less than one standard deviation. (A smaller $\chi^{2}$ is not necessarily better in a $\chi^{2}$ test: the best fit occurs when $\chi^{2}=v$.) The qualities of the SNe Ia fits (ours and the Planck team's) are commensurate, but the team's $\Lambda$ CDM model depends on the existence of an untenable fiction: dark energy. 
The $\Lambda \mathrm{CDM}$ model is based on Equation (14) (but while also implicitly ignoring Equation (15)). The first integral of Equation (14) is

$$
2 \frac{\mathrm{d} \phi}{\mathrm{d} r}=\frac{(8 \pi k \varrho-\Lambda) r}{3},
$$

which, by Newton's second law of motion, is

$$
\frac{\mathrm{d}^{2} r}{\mathrm{~d} t^{2}}=\frac{r}{6}(\Lambda-8 \pi k \varrho) .
$$

With this choice, the $\Lambda$ CDM scale factor for an expanding universe decelerates at first when $8 \pi k \varrho>\Lambda$, then later accelerates after $\Lambda>8 \pi k \varrho$; the orthodox $\Lambda \mathrm{CDM}$ model does not expand at the same rate as the well substantiated linearly coasting universe that are discussed in Section 4 . There is no way of formulating a linearly coasting universe with the $\Lambda \mathrm{CDM}$ model, so purported $\Lambda \mathrm{CDM}$ scale accelerations [60] [63]-[68] are then merely expected artifacts of the Equation (17) procrustean template. Anyway, according to Equation (9) of Section 3, and to the conventionally ignored Equation (15), the cosmological constant must be zero: the $\Lambda \mathrm{CDM}$ model should be rejected because it does not expand at all.

\section{Conclusions}

GR can be reconciled with a static universe by starting with the Einstein field equation (Equation (1)) if $p=0$, or with the modified field equation (Equation (4)) if $p \geq 0$. GR can also be reconciled with a uniformly expanding universe by replacing the Einstein tensor with its Lovelock form $\boldsymbol{G}_{\mu v} \rightarrow \boldsymbol{G}_{\mu v}+b \boldsymbol{g}_{\mu v}$. GR then turns out to be in full accord with the linear coasting universe determined from the collation of type Ia supernovae ( $\mathrm{SNe}$ Ia) brightness and redshift data-without recourse to the existence of dark matter or dark energy.

The $\Lambda C D M$ model requires the existence of vast quantities of (attractive) dark matter and (repulsive) dark energy. They are the sources of fanciful opposing forces that mostly cancel each other out. We have shown why this model must be wrong: according to Equation (15), $\Lambda=0$. There can be no dark energy in the context of the $\Lambda \mathrm{CDM}$ approach, so this broadly accepted model is incorrect. Whereas dark energy is implausible and unnecessary, dark matter is not implausible and its need is unresolved. We recommend a linearly coasting universe-one that is consistent both with GR and with SNe Ia observations-as a viable $\Lambda \mathrm{CDM}$ alternative.

\section{Conflicts of Interest}

The authors declare no conflicts of interest regarding the publication of this paper.

\section{References}

[1] Baum, R. and Sheehan, W. (1997) Search of Planet Vulcan. Plenum Press, New York. https://doi.org/10.1007/978-1-4899-6100-6

[2] Aprile, R., et al. (2018) Physical Review Letters, 121, Article ID: 111302. 
https://doi.org/10.1103/PhysRevLett.121.111302

[3] Agnes, P., et al. (2018) Physical Review Letters, 121, Article ID: 081307. https://doi.org/10.1103/PhysRevLett.121.081307

[4] Albert, A., et al. (2017) The Astrophysical Journal, 834, 110. https://doi.org/10.3847/1538-4357/834/2/110

[5] Kahlhoefer, F. (2017) International Journal of Modern Physics A, 32, Article ID: 1730006. https://doi.org/10.1142/S0217751X1730006X

[6] Hoof, S., Geringer-Sameth, A. and Trotta, R. (2020) Journal of Cosmology and Astroparticle Physics, No. 2, 012. https://doi.org/10.1088/1475-7516/2020/02/012

[7] Hales, S.D. (2005) Think, 4, 109. https://doi.org/10.1017/S1477175600001287

[8] Kang, Y., Lee, Y.-W., Kim, Y.-L., Chung, C. and Ree, C.H. (2020) The Astrophysical Journal, 889, 8. https://doi.org/10.3847/1538-4357/ab5afc

[9] Kenyon, I.R. (1990) General Relativity. Oxford University Press, Oxford.

[10] Peebles, P.J.E. (1993) Principles of Physical Cosmology. Princeton University Press, Princeton.

[11] Einstein, A. (1923) The Meaning of Relativity. Princeton University Press, Princeton.

[12] Genova, A., et al. (2018) Nature Communications, 9, 289. https://doi.org/10.1038/s41467-017-02558-1

[13] Wolfram, S. (1999) The Mathematica Book. Cambridge University Press, Cambridge.

[14] Hartle, J.B. (2002) Gravity: An Introduction to Einstein's General Relativity. Addison-Wesley Pub. Co., Boston.

[15] Lovelock, D.J. (1971) Journal of Mathematical Physics, 12, 498. https://doi.org/10.1063/1.1665613

[16] Milgrom, M. (1983) The Astrophysical Journal, 270, 365. https://doi.org/10.1086/161130

[17] Milgrom, M. (1983) The Astrophysical Journal, 270, 371. https://doi.org/10.1086/161131

[18] Brownstein, J.R. and Moffat, J.W. (2006) The Astrophysical Journal, 636, 721. https://doi.org/10.1086/498208

[19] Mannheim, P.D. and O’Brien, J.G. (2011) Physical Review Letters, 106, Article ID: 121101. https://doi.org/10.1103/PhysRevLett.106.121101

[20] Verlinde, E.P. (2017) SciPost Physics, 2, 016. https://doi.org/10.21468/SciPostPhys.2.3.016

[21] Pestaña, J.L.G. and Eckhardt, D.H. (2011) The Astrophysical Journal Letters, 741, L31. https://doi.org/10.1088/2041-8205/741/2/L31

[22] McGaugh, S. (1999) The Astrophysical Journal Letters, 523, L99. https://doi.org/10.1086/312274

[23] McGaugh, S. (2000) The Astrophysical Journal Letters, 541, L33. https://doi.org/10.1086/312902

[24] McGaugh, S. (2004) The Astrophysical Journal, 611, 26. https://doi.org/10.1086/421895

[25] Angus, G. and Diaferio, A. (2011) MNRAS, 417, 941. https://doi.org/10.1111/j.1365-2966.2011.19321.x

[26] Bahcall, N., Ostriker, J.P., Perlmutter, S. and Steinhardt, P.J. (1999) Science, 284, 
1481. https://doi.org/10.1126/science.284.5419.1481

[27] Kolb, E.W. (1989) The Astrophysical Journal, 344, 543. https://doi.org/10.1086/167825

[28] Sethi, G., Dev, A. and Jain, D. (2005) Physics Letters B, 624, 135. https://doi.org/10.1016/j.physletb.2005.08.005

[29] John, M.V. and Narlikar, J.V. (2002) Physical Review D, 65, Article ID: 043506. https://doi.org/10.1103/PhysRevD.65.043506

[30] Dev, A., et al. (2002) Physics Letters B, 548, 12. https://doi.org/10.1016/S0370-2693(02)02814-9

[31] John, M.V. (2005) The Astrophysical Journal, 630, 667. https://doi.org/10.1086/432111

[32] Zhu, Z.-H., et al. (2008) Astronomy \& Astrophysics, 483, 15. https://doi.org/10.1051/0004-6361:20077797

[33] Eckhardt, D.H., Pestaña, J.L.G. and Fischbach, E. (2010) New Astronomy, 15, 175. https://doi.org/10.1016/j.newast.2009.07.005

[34] Bilicki, M. and Seikel, M. (2012) Monthly Notices of the Royal Astronomical Society, 425, 1664. https://doi.org/10.1111/j.1365-2966.2012.21575.x

[35] Melia, F. and Shevchuk, A.S.H. (2012) Monthly Notices of the Royal Astronomical Society, 419, 2579. https://doi.org/10.1111/j.1365-2966.2011.19906.x

[36] Mitra, M. (2014) Monthly Notices of the Royal Astronomical Society, 442, 382. https://doi.org/10.1093/mnras/stu859

[37] López-Corredoira, M. (2014) The Astrophysical Journal, 781, 96. https://doi.org/10.1088/0004-637X/781/2/96

[38] Melia, F. (2014) Astronomy \& Astrophysics, 561, A80. https://doi.org/10.1051/0004-6361/201322285

[39] Melia, F. and McClintock, T.M. (2015) The Astronomical Journal, 150, 119. https://doi.org/10.1088/0004-6256/150/4/119

[40] Shafer, D.L. (2015) Physical Review D, 91, Article ID: 103516. https://doi.org/10.1103/PhysRevD.91.103516

[41] Singh, P. and Lohiya, D. (2015) Journal of Cosmology and Astroparticle Physics, 5, 061. https://doi.org/10.1088/1475-7516/2015/05/061

[42] Wei, J.-J., Wu, X.F. and Melia, F. (2015) The Astronomical Journal, 149, 165. https://doi.org/10.1088/0004-6256/149/5/165

[43] Lopez-Corredoira, M., et al. (2016) International Journal of Modern Physics D, 25, Article ID: 1650060. https://doi.org/10.1142/S0218271816500607

[44] Melia, F. (2016) Proceedings of the Royal Society A, 472, Article ID: 20150765. https://doi.org/10.1098/rspa.2015.0765

[45] Melia, F. (2016) Monthly Notices of the Royal Astronomical Society, 463, L61. https://doi.org/10.1093/mnrasl/slw157

[46] Nielsen, J.T., Guffanti, A. and Sarkar, S. (2016) Scientific Reports, 6, Article No. 35596. https://doi.org/10.1038/srep35596

[47] Tutusaus, I., et al. (2016) Physical Review D, 94, Article ID: 103511. https://doi.org/10.1103/PhysRevD.94.103511

[48] Wei, J.-J., Wu, X.F. and Melia, F. (2016) Monthly Notices of the Royal Astronomical Society, 463, 1144. https://doi.org/10.1093/mnras/stw2057

[49] Zeng, H., Melia, F. and Zhang, L. (2016) Monthly Notices of the Royal Astronomi- 
cal Society, 462, 3094. https://doi.org/10.1093/mnras/stw1817

[50] Tutusaus, I., et al. (2017) Astronomy \& Astrophysics, 602, A73. https://doi.org/10.1051/0004-6361/201630289

[51] Melia, F. (2017) Monthly Notices of the Royal Astronomical Society, 464, 1966. https://doi.org/10.1093/mnras/stw2493

[52] Melia, F. and López-Corredoira, M. (2017) International Journal of Modern Physics $D$, 26, Article ID: 1750055. https://doi.org/10.1142/S0218271817500559

[53] Wei, J.-J., Melia, F. and Wu, X. (2017) The Astrophysical Journal, 835, 270. https://doi.org/10.3847/1538-4357/835/2/270

[54] Leaf, K. and Melia, F. (2018) Monthly Notices of the Royal Astronomical Society, 474, 4507. https://doi.org/10.1093/mnras/stx3109

[55] Yennapureddy, M.K. and Melia, F. (2018) Physics of the Dark Universe, 20, 65. https://doi.org/10.1016/j.dark.2018.03.003

[56] Melia, F., et al. (2018) Europhysics Letters, 123, 59002. https://doi.org/10.1209/0295-5075/123/59002

[57] Melia, F. (2019) Monthly Notices of the Royal Astronomical Society, 489, 517. https://doi.org/10.1093/mnras/stz2120

[58] John, M.V. (2019) Monthly Notices of the Royal Astronomical Society, 484, L35. https://doi.org/10.1093/mnrasl/sly243

[59] Dolgov, A.D., Sazhin, M.V. and Zeldovich, Y.B. (1990) Basics of Modern Cosmology. Editions Frontières, Gif-sur-Yvette, France.

[60] Suzuki, N., et al. (2012) The Astrophysical Journal, 746, 85. https://doi.org/10.1088/0004-637X/746/1/85

[61] Ade, P.A.R., et al. (2014) Astronomy \& Astrophysics, 571, A16. https://doi.org/10.1051/0004-6361/201321591

[62] Bond, H.E., et al. (2013) The Astrophysical Journal Letters, 765, L12. https://doi.org/10.1088/2041-8205/765/1/L12

[63] Riess, A.G., et al. (1998) The Astronomical Journal, 116, 1009. https://doi.org/10.1086/300499

[64] Perlmutter, S., et al. (1999) The Astrophysical Journal, 517, 565. https://doi.org/10.1086/307221

[65] Riess, A.G., et al. (2004) The Astrophysical Journal, 607, 665. https://doi.org/10.1086/383612

[66] Riess, A.G., et al. (2007) The Astrophysical Journal, 659, 98. https://doi.org/10.1086/510378

[67] Frieman, J.A., Turner, M.S. and Huterer, D. (2010) Annual Review of Astronomy and Astrophysics, 46, 385. https://doi.org/10.1146/annurev.astro.46.060407.145243

[68] Amanullah, R., et al. (2010) The Astrophysical Journal, 716, 712. https://doi.org/10.1088/0004-637X/716/1/712 